ICNMM2008-62385

\title{
ON THE RESILIENCE OF PDMS MICROCHANNELS AFTER VIOLENT OPTICAL BREAKDOWN MICROBUBBLE CAVITATION
}

\author{
D. Fernandez Rivas* \\ d.fernandezrivas@tnw.utwente.nl \\ Mesoscale Chemical Systems Group \\ MESA+ Research Institute, University of Twente \\ Meander ME147, POBox 217 | 7500AE Enschede The Netherlands
}

\begin{abstract}
The use of the silicone known as Poly-dimethylsiloxane (PDMS) in microfluidic and other activities has increased enormously. By means of a laser short pulse, optical breakdown of the liquid contained in a microchannel (squared cross-section with $100 \mu \mathrm{m}$ sides) can occur; as a consequence plasma is generated and a gaseous bubble suddenly expands. The expansion of such a bubble can be so violent that the channel walls deform considerably and in some cases the detachment of the top wall from the PDMS channel is verified. Measurements of channel width reveal a relative increase up to $45 \%$ for the tested conditions. Due to the elasticity of PDMS and surface energy values, surprisingly the top wall and channel reattach. Contrary to widely known cavitation where damage to the wall is manifested in form of pitting, we observe no permanent damage for the experimental conditions studied. We envisage the use of PDMS material in other types of applications with high relative volumetric change, such as microexplosions, ultrasonic cavitation and the like, in which there is a cyclic stress load of the PDMS material. We present a compact method to predict the reliability of PDMS for its use in cavitational events occurring inside of microfluidic channels (by force balance and energy conservation analysis).
\end{abstract}

\section{INTRODUCTION}

The use of laser pulses is a very reproducible way to generate bubbles and it is known as Laser-induced optical breakdown (LOB). The absorbed energy leads to plasma formation and a certain amount of liquid is evaporated forming a gaseous bubble which expands and subsequently collapses. Vast literature covers this phenomenon from different perspectives (medical, cleansing and other types of applications). The work of Vogel and collaborators [1] gives a detailed explanation of the conversion of laser energy during optical breakdown at different laser energies and pulse duration. The high conversion efficiency into mechanical energy is the cause of the disruptive character of the plasma formation (e.g. laser surgery and micromachining in transparent liquid materials. Most published works relate the formation of a bubble either in a liquid vessel where the effects of the walls can be neglected, or near a rigid or flexible wall. Little attention has been paid to closed geometries with boundaries near to the generated bubble. The recorded cavitation events in our experiments have been observed before [2] but their aim was to increase the mixing of two parallel flowing streams in microchannels with similar geometry. No interest to the dynamic loading conditions (channel walls deformation by high pressure expanding bubble) inside the microchannel is given there.

\subsection{OPTICAL BREAKDOWN ENERGY BALANCE}

For the operated laser conditions, from [1] we get the following table:

Table 1. Energy balance for laser shot configuration (values are taken from [1])

\begin{tabular}{ccccccc}
$\begin{array}{c}\text { Wavelength } \\
{[\mathrm{nm}]}\end{array}$ & $\begin{array}{c}\text { Pulse } \\
\text { duration } \\
{[\mathrm{ns}]}\end{array}$ & $\begin{array}{c}\text { Energy [J] } \\
\mathrm{E}_{\mathrm{in}} \rightarrow 100 \%\end{array}$ & Radiated & ScatteredTransmittedAbsorbed \\
\hline 532 & 6 & $1,0 \mathrm{E}-03$ & $0.8 \%$ & $0.5 \%$ & $8.1 \%$ & $26,50 \%$ \\
\hline $\begin{array}{c}\text { Converted } \\
\text { Energy }\end{array}$ & $\begin{array}{c}\text { Shock } \\
\text { wave }\end{array}$ & Bubble & Evaporation $\begin{array}{c}\text { Plasma } \\
\text { radiation }\end{array}$ & Difference & \\
\hline$\%$ of $\mathrm{E}_{\text {in }}$ & $36.4 / 58.3$ & 24.9 & 7.5 & $6 \times 10-4$ & 10
\end{tabular}

It is clear that most of the energy goes into evaporation, shock wave generation and cavitation.

\footnotetext{
${ }^{*}$ Author of correspondence
} 


\subsection{PDMS DEFFORMATION AND EROSION}

From the literature of Materials Resistance [3], we follow Generalized Hook's Law and find that the stress $\sigma$ following the "thin spring" approach [4] can be calculated as:

$\sigma=E \varepsilon=E\left(\frac{l-a}{a}\right)$

The deformations in a material can be considered elastic if the inner pressures $\mathrm{P}$ are smaller than half the fracture or tensile strength

$P<\sigma_{f r} / 2$

In [5] the deformation at the center of a semi-infinite body $b$ due to a loading pressure applied on a circular region of radius $\mathrm{R}$ is given by

$b(r=0)=\frac{2\left(1-\mu^{2}\right) P R}{E}$

It is suggested for the case of a bubble near an elastic boundary that the loading region radius can be the same as the bubble radius. From the deformed area value, the work done by the expanding bubble is stored as energy given by:

$U_{\text {Fbalance }}=\frac{1}{2} P * b *$ Area

Interesting references of the effects of cavitating bubbles of different materials have been published elsewhere $[6,7,8]$. Nevertheless, little or no attention has been paid to find if PDMS can be damaged in a similar way as ship propellers, on the contrary the study of biological materials have been analyzed such as cornea tissue. An important feature to keep in mind is that most previous publications of the dynamics of laser induced cavitation bubbles near either solid or elastic boundaries consider one infinite wall, we have four walls.

\section{EXPERIMENTAL}

Zwaan and collaborators have presented the equipment used in this study [9]. Each cavitation event is captured using a fast camera (HPV-1, Shimadzu Corp. Kyoto, Japan) with up to $1 * 10^{6}$ frames per second (fps).

Standard photolithographic procedures were performed to obtain channels with squared cross-section of $100 \mu \mathrm{m}$ side. The PDMS polymer and curing agent (Sylgard 184, Dow Corning Corp.) are mixed in a 10:1 ratio by weight. The PDMS mixture was poured over a SU-8 mold with the necessary structure's size and baked at $70{ }^{\circ} \mathrm{C}$ for one hour. After peeling off, plasma bonding to microscope glass slides was done.

The mechanical properties of polymeric materials employed in microfluidic applications such as polyacrilamide (PAA) and PDMS have been reported $[8,10,11]$. In the case of PDMS, different values of the elasticity modulus E, also known as Young's Modulus, and shear modulus G can be achieved. The most widely used mixing ratio is 10:1 and the reported values are $\mathrm{E}=0.75 \mathrm{MPa}$ and $\mathrm{G}=0.25 \mathrm{MPa}$, making it the most flexible polymer. The relation of $\mathrm{E}$ and $\mathrm{G}$ comes from:

$$
E=2 G(\mu+1)
$$

Where $\mu$ is the Poisson coefficient, with a value of 0.5 i.e. incompressible materials (a strain will not change its volume) and the fracture or tensile strength $\sigma_{f r}=2.24 \mathrm{MPa}$ [12].

Microfluidic chips were filled with distilled water and after the laser was aligned at the center of the PDMS channel, the experiment was conducted. Deformed and deformed wall length ( $a$ and 1 respectively) was measured as well as the expansion of the channel width $\mathrm{W}$; the relative channel width expansion s was calculated as:

$s=\frac{W^{\prime}-W}{W} \%$

In a second series of experiments, water with food dye was used to increase energy absorbance from the laser, ensuring more violent cavitation events. Pointing to different positions, each time a sequence of laser shots $(100,200,300$ and 500 times) was programmed with varying energy $(0.2,0.4,0.8$ and $1 \mathrm{~mJ}$ ). Finally, the laser focus was set to the surface of the channel to record the damage caused by the direct impact of laser in the PDMS channel wall. Surfaces of interest were inspected with a microscope as well as Scanning Electron Microscopy (SEM) analysis.

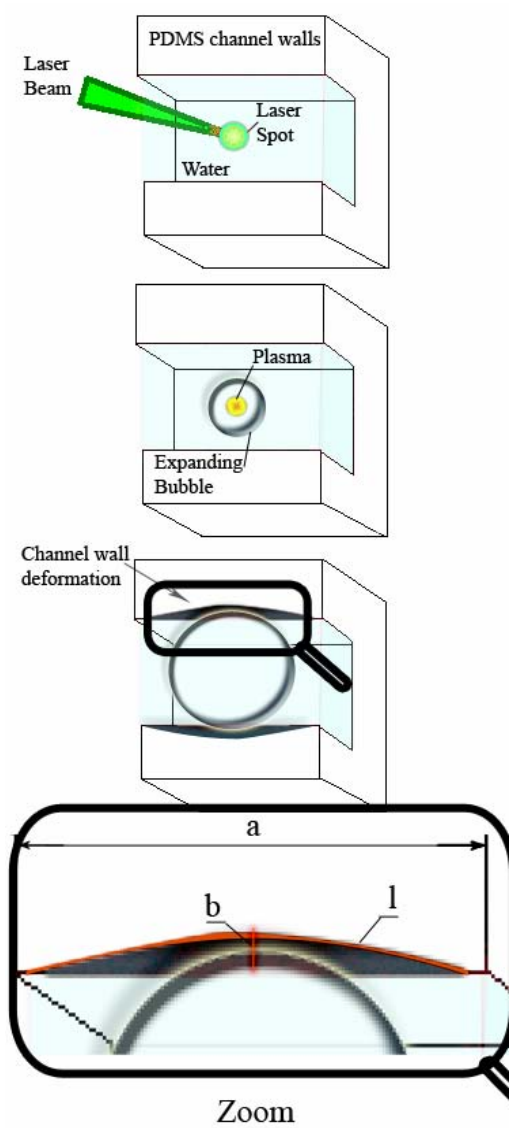

Figure 1. Schematic representation of the experiment conditions. 


\section{3}

\section{RESULTS}

In next figure, a sequence of captured images with $63000 \mathrm{fps}$ of the dynamic evolution of a cavitating bubble is depicted.

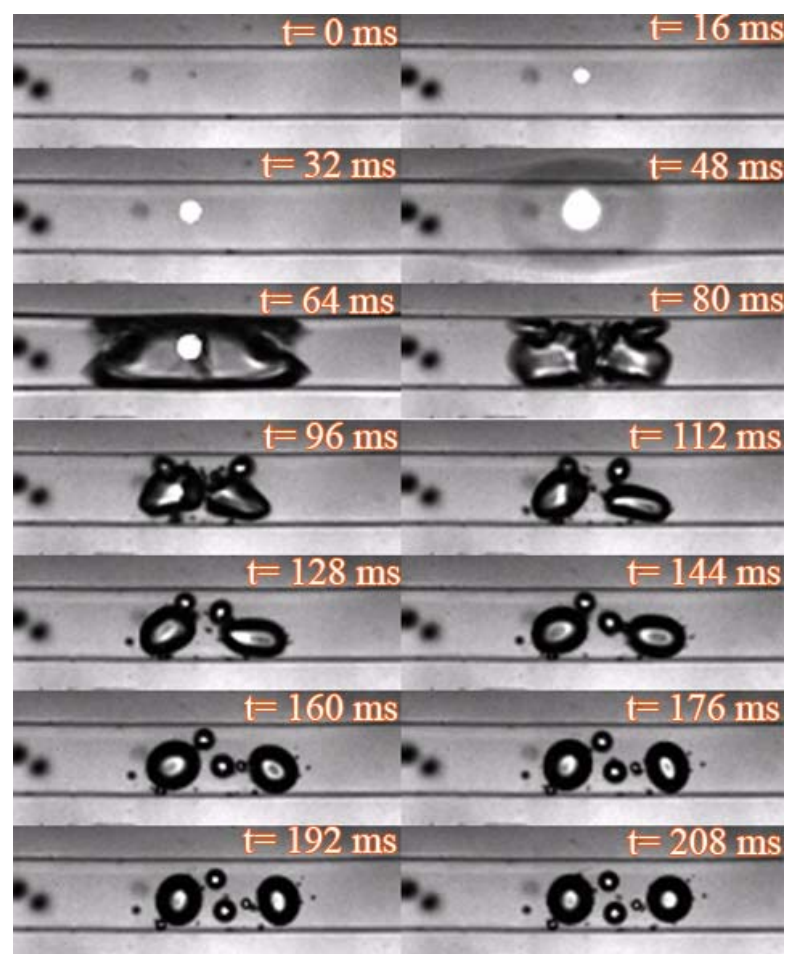

Figure 2. Sequence of different moments in the optical breakdown inside a squared cross-section microchannel. Time difference between each snapshot is $16 \mu \mathrm{s}$. Order is up-down, from left to right.

\subsection{MEASURED DEFORMATION VALUES}

The frame in which the maximum expansion of the channel walls was reached was analyzed and several parameters were measured.

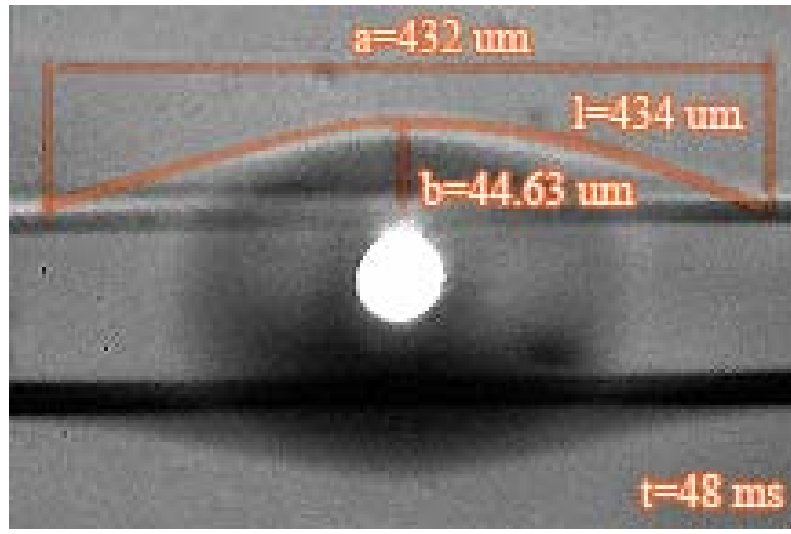

Figure 3. Maximum expansion of the channel width.

A selection of three experimental conditions was averaged and the values are presented in the table given bellow.
Table 2. Measured values

\begin{tabular}{cccc}
\hline Experiment situation & 1 & 2 & 3 \\
\hline Laser Energy $\mathrm{E}_{\text {in }}[\mathrm{J}]$ & $8,0 \mathrm{E}-04$ & $1,2 \mathrm{E}-03$ & $1,5 \mathrm{E}-03$ \\
$\mathrm{~A}[\mu \mathrm{m}]$ & 532 & 328 & 432 \\
$\mathrm{~b}[\mu \mathrm{m}]$ & 26,5 & 37 & 44,63 \\
$1[\mu \mathrm{m}]$ & 534,2 & 331,0 & 433,5 \\
$\begin{array}{c}\text { Relative expansion } \\
\mathrm{s}[\%]\end{array}$ & $26,5 \%$ & $37,0 \%$ & $44,6 \%$ \\
$\begin{array}{c}\text { Optical breakdown } \\
\text { energy balance }[\mathrm{J}]\end{array}$ & $1,8 \mathrm{E}-07$ & $2,7 \mathrm{E}-07$ & $3,4 \mathrm{E}-07$ \\
$\begin{array}{c}\text { Force balance energy } \\
\text { value }[\mathrm{J}]\end{array}$ & $1,9 \mathrm{E}-07$ & $2,3 \mathrm{E}-07$ & $4,3 \mathrm{E}-07$
\end{tabular}

The comparison between both methods shows acceptable resemblance qualitatively and the calculated and measured values fall quite close from each other. A plot of these values is given in next figure:

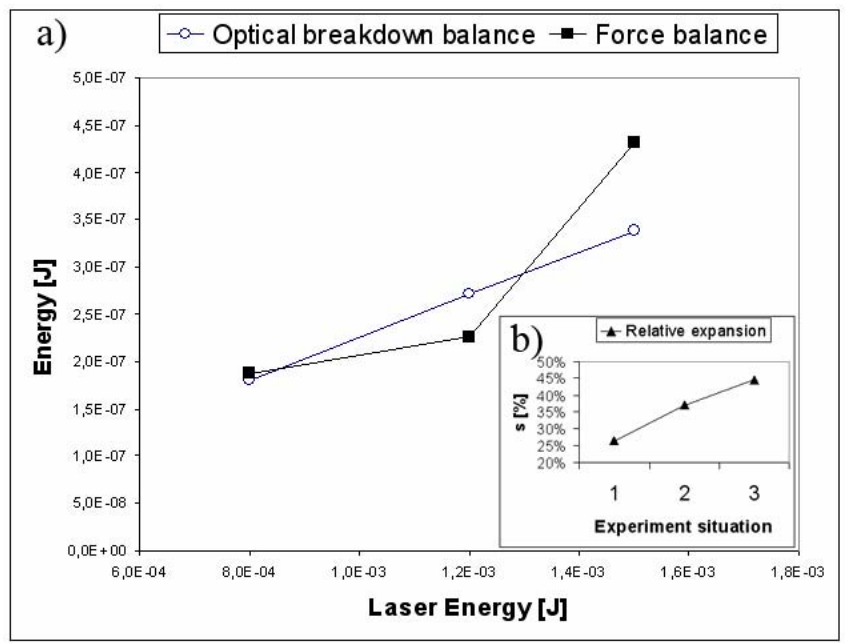

Figure 4. a) Compared plot of the expected values from optical breakdown approach and calculated from experimental measurements.

b) Relative expansion of channel width for each case.

\subsection{RESULTS DISCUSSION}

By observing the image sequence in Hellman's work [2] and ours, it can be seen that the shock wave travels faster than the expansion of the bubble wall (shock wave trespassed the wall in the first $50 \mathrm{~ns}$, and the bubble surface gets close to the channel wall in approximately 0.24 to $4 \mu \mathrm{s}$ ). This means that the shock wave does not influence the maximum deformation of the channel's walls.

The collapse of the bubble commences just after the biggest bubble size is reached. Due to the influence of the four channel walls, the deformed elipsoidal bubble starts splitting in four smaller bubbles as a result of jetting towards the walls.

Neither optical inspection with a microscope or SEM analysis revealed any damage of the PDMS surface at any point except from the direct impact on channel wall (see Figure 5). This suggests that besides the known advantages of PDMS in microfluidic applications (optical transparency, biocompatibility and the like), one can think of other novel applications to name: chemical reactions with increased 
volume, microexplosions, repeated cavitation with a specific purpose (e.g. water treatment by means of ultrasonic treatment could be carried as PDMS is an excellent medium for ultrasonic wave [13]).

Besides the study of bubble expansion and collapse in confined geometries, LOB can be used to find the response time of channel material or for combining it with other applications such as ultrasound cavitation and hydrodynamic cavitation events. The behavior of PDMS proved to be resistant to high stresses and deformations at such small scales, which could make it suitable than silicon or other materials for explosive reactions. Another interesting feature of our experiments is that the bonding of PDMS to glass endured the cyclic overpressure conditions. In some cases there is a momentary detachment, but the surfaces reattach.

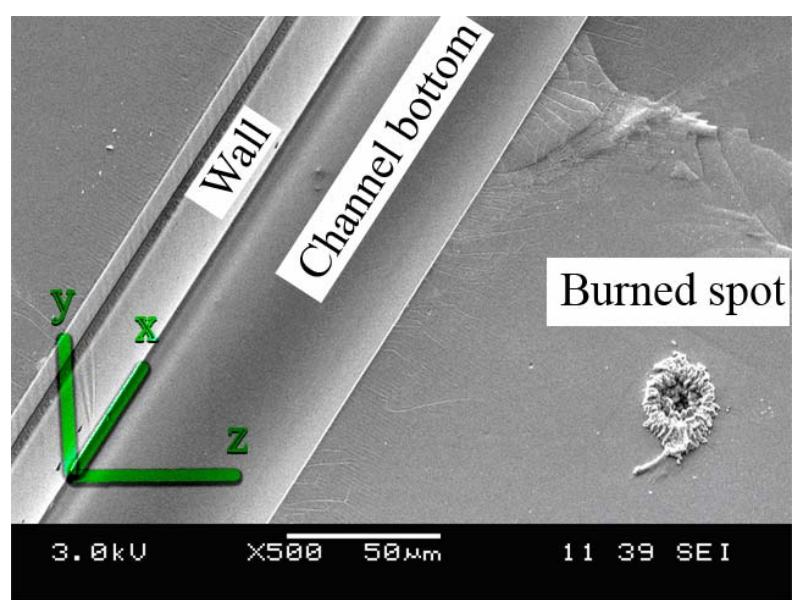

Figure 5. Channel section from a bird's view and to the side the effect of laser shot focused at the PDMS surface.

The most dramatic scenario to which our chips were exposed (500 times laser shots with energy equal to $1 \mathrm{~mJ}$ ) allow us to stress that PDMS chips are suitable for extreme conditions reactions. If the pressure inside the channel is bellow $1.12 \mathrm{MPa}$, the deformation of PDMS should behave elastic, and no plastic deformation should occur. This pressure not only refers to the inner pressure of the bubble, but also to the one given by the impact of the jets. The energy of the laser was not increased in the aim to reach the resistance or fracture strength limit $\left(\mathrm{P}>\sigma_{\mathrm{fr}}\right.$, with the consequent effect of pitting the channel surface) to avoid damaging the CCD chip of the fast camera due to scattered light.

\section{CONCLUSIONS}

The experimental evidence shows that PDMS could be used for the fabrication of microfluidic chips to conduct chemical reactions with marked increased volume, microexplossions and repeated cavitation with a specific purpose such as water treatment, for fine chemical production or even sonochemistry. Contrary to other materials exposed to bubble cavitation events, pitting of the surface was not observed in our experiments.

Experimental measurements of the fatigue limit of PDMS would be of relevance in future works.

\section{$5 \quad$ ACKNOWLEDGMENTS}

This work has been funded by STW Project Number 07391 (The Netherlands).

The support of the Physics of Fluids group at Twente University was fundamental for the conduction of the experimental work. Discussions with C. Sun, R.J. Dijkink, M. Versluis and D. Lohse are highly appreciated.

\section{REFERENCES}

A. Vogel, J. Noack, K. Nahen, D.Theisen, S. Busch, U. Parlitz, D.X Hammer, G.D. Noojin, B.A. Rockwell and R. Birngruber, Appl. Phys. B, 1999, 68, 271-280.

2 A.N. Hellman, K.R. Rau, H.H. Yoon, S. Bae, J. F. Palmer, K.S. Phillips, N.L. Allbritton and V. Venugopalan, Anal. Chem., 2007, 79, 4484-4492.

V.I. Feodosiev, in Resistencia de Materiales, MIR, Moscow, $3^{\text {rd }}$ edn., 1985 , ch. 7, pp. 268-281.

4 E.P. Kartalov, A. Scherer, S.R. Quake, C.R. Taylor and W.F. Anderson, J. Appl. Phys., 2007, 101, 064505.

E.Klaseboer, B.C. Khoo, J. Appl. Phys., 2004, 96, No. 10, pp. 5808-5818

6 T.G. Leighton, in The Acoustic Bubble, Academic Press, London, 1994, pp. 531-551.

A. Vogel, W. Lauterborn and R. Timm, J. Fluid Mech., 1989, 2006, pp. 299-338

8 E.A. Brujan, K. Nahen, P. Schmidt and A. Vogel, J. Fluid Mech., 2001, 433, pp. 251-281.

9 E. Zwaan, S. le Gac, K. Tsuji and C.D. Ohl, PRL, 2007, 98, 254501.

10 D. Armani, C. Liu and N. Aluru, Proc. Twelfth IEEE International Conference on Micro Electro Mechanical Systems, 1999, pp.222-227.

11 J.C. Loetters, W. Olthuis, P.H. Veltink and P. Bergveld, J. Micromech. Microeng., 1997, 7, pp. 145-147.

12 J. Mark, in Polymer Data Handbook, Oxford Univ. Press, New York 1999.

13 Y. Iida, T. Tuziuti, K. Yasui, A. Towata and T. Kozuka, Ultrasonics Sonochemistry $2007, \mathbf{1 4}$, pp. 621-626 